\title{
Conservation laws and theorems of confinement and stability for a charged equatorial disk in a pulsar magnetosphere
}

\author{
J. J. Aly
}

\author{
CEA/DSM/DAPNIA, Service d'Astrophysique (CNRS FRE 2591), CE Saclay, 91191 Gif-sur-Yvette Cedex, France \\ e-mail: jjaly@discovery.saclay.cea.fr
}

Received 21 July 2004 / Accepted 14 December 2004

\begin{abstract}
For studying the nonaxisymmetric stability of the bounded electrosphere of an "aligned pulsar" (Michel's structure with polar domes and equatorial belt), Pétri et al. (2002) recently introduced a simplified but useful model in which all the charge-separated plasma located outside the magnetized rotating star is concentrated into a thin equatorial disk. In this paper, some aspects of this model are investigated analytically. It is shown that the equations governing the behaviour of the disk - in the case where there are no sources of particles feeding it - imply a series of conservation laws (for energy, angular momentum,...), and that there is a non-canonical Hamiltonian structure hidden behind them. The conservation laws are used to prove that: (i) for any initial conditions imposed on the disk, its evolution cannot lead to charges escaping to infinity (confinement theorem); (ii) a disk steady state with a possibly rotating pattern is nonlinearly stable if the charge density per unit of magnetic flux is a decreasing function of the electrostatic potential in the rotating frame (stability theorem).
\end{abstract}

Key words. pulsars: general - magnetic fields - plasmas

\section{Introduction}

The structure of the magnetosphere around an isolated rotating neutron star has been the object of intense investigations since the discovery of pulsars. In the axisymmetric aligned case, a beautiful solution to that problem has been proposed by Michel (1980). On the basis of general physical arguments, Michel conjectured that the magnetosphere (or the electrosphere in his terminology) has a finite extent, and is constituted of two charged domes overlying the poles and of a charged equatorial belt. Later, this picture was confirmed by numerical simulations, first by Krause-Polstorff \& Michel (1985a,b), and most recently, with an improved resolution, by Smith et al. (2001), Pétri et al. (2002a) and Spitkovsky \& Arons (2002).

However, this may not be the end of the story. Indeed, Pétri et al. (2002b, 2003) and Spitkovsky \& Arons (2002) have found Michel's configuration to be subject to a specific nonaxisymmetric instability of the diocotron type (see, e.g., Davidson 1990). The latter develops in the equatorial belt, which is connected to the star by magnetic lines threading vacuum regions. Thus the plasma therein is allowed to rotate differentially at equilibrium - this is the source of the instability - and to suffer interchange motions when it is perturbed.

To study this instability, Petri et al. $(2002 \mathrm{~b}, 2003)$ introduced a simplified picture in which the equatorial belt is taken to be an infinitesimally thin disk, the presence of the domes is not taken into account, and plasma moves at the drift velocity. It is the aim of this paper to analytically investigate a few aspects of this model in the case where there are no sources of charges - extraction from the star, pair creation,... - feeding the disk. More precisely, we are interested in exploring the structure of the full set of nonlinear equations governing the evolution of the system. In particular, we want to exhibit the conservation laws which are embodied into them, and derive some of their possible general consequences: (i) for the degree of confinement of the particles by the magnetic field when the disk evolves from some arbitrarily fixed initial state; (ii) for the stability of a steady state.

Actually, an analytical study of the disk model has already been undertaken in Pétri et al. (2003), which we read after completion of this work. In it angular momentum conservation is discussed and a confinement theorem is established. We also note that questions similar to those we are interested in have long been addressed by physicists working on laboratory non-neutral plasmas. But the results which have been obtained in the latter framework, although providing useful guidelines, cannot be immediately transposed to our situation, as they usually deal with plasmas enclosed in rigid vessels and radially confined either by a uniform magnetic field (Davidson 1990; Davidson \& Lund 1991; O’Neil 1980) or by a purely toroidal one (O’Neil \& Smith 1994).

The paper is organized as follows. We first give a detailed description of the model (Sect. 2) and establish a few useful formulas for the electrostatic potential and field (Sect. 3). Next, in Sect. 4, we put the equations into a most convenient form by effecting a change of variables, and we note that there is a non-canonical Hamiltonian structure hidden behind them. 
In Sect. 5 we prove that during the evolution of the system, there is conservation of a series of global quantities: an infinite family of functionals of the charge density per unit of magnetic flux in addition to angular momentum and energy. Note that the derivation of these laws could have been done in a most elegant way in the framework of the non-canonical description alluded to above. However, some readers may be unfamiliar with this abstract formulation, and we found it preferable to keep in that section (and the following ones) to a more traditional approach (consequently, Sect. 4.3 and the related Appendix A can be skipped without any inconvenience by the uninterested reader). In Sect. 6, we establish two confinement theorems, and we give in Sect. 7 the general form of the steady states (possibly rotating) and a sufficient condition for them to be stable. Finally (Sect. 8), we summarize our results and compare them to those already known for laboratory charged plasmas, and we briefly discuss their possible consequences for the modeling of pulsar magnetospheres.

\section{Description of the model and equations}

We start by giving a detailed description of the model, referring to Pétri et al. (2002b, 2003) for justifications of its validity and usefulness.

\subsection{Pulsar, disk and magnetic field}

In the model considered in this paper, the pulsar occupies a spherical domain $D_{*}$ with center $O$ and radius $r_{*}$, bounded by the surface $S$. Its external layers are constituted of a perfectly conducting material, and it rotates at the constant angular velocity $\Omega_{*} \hat{z}$ (we use Cartesian coordinates $(x, y, z)$ centered at $O$ and associated spherical coordinates $(r, \theta, \varphi))$. Inside the pulsar, there are intrinsic distributions of electric currents and electric charges which are constant (in time), axisymmetric, and mirrorsymmetric (with respect to the plane $\{\theta=\pi / 2\}$ ).

The region of space $D$ outside $D_{*}$ is a vacuum, except for an infinitesimally thin disk $\Sigma$ of charge-separated plasma lying in the equatorial domain $P=\left\{r>r_{*}, \theta=\pi / 2\right\}$. The electric charge on $\Sigma$ is distributed with a surface density $\sigma(r, \varphi, t)$ (note the possible dependence on the azimuthal angle $\varphi$ and the time $t$ ), and we agree that $\sigma$ is defined over the whole $P$, with $\sigma=0$ outside of $\Sigma$. We assume that there are neither sources nor sinks able to exchange charges with the disk.

The magnetic field $\boldsymbol{B}$ is created by the only currents flowing inside the pulsar, i.e., we neglect the contribution arising from the motion of the charges in $\Sigma$ (this is justified as long as their velocities stay small compared to the speed of light $c$ ). Thus, in $D, \boldsymbol{B}$ is potential, constant, axisymmetric and $z$-symmetric, implying that $\boldsymbol{B}=B_{\theta} \hat{\boldsymbol{\theta}}$ on $P$. The exact form of $\boldsymbol{B}$ need not be fixed in a precise way for the following discussion. In any case, $\boldsymbol{B}$ has a dipolar component of moment $m_{*} \hat{z}$, but higher order contributions may also be present. We just assume that $B_{\theta}$ does not vanish in $P$, and without loss of generality we impose $B_{\theta}=B>0$ (therefore, $m_{*}>0$ ). Of course,

$B(r, \pi / 2) \leq m / r^{3}$ with $m$ some positive number (when $\boldsymbol{B}$ is purely dipolar, we merely have $\left.B(r, \pi / 2)=m_{*} / r^{3}\right)$. Finally, we recall the usual representation

$\boldsymbol{B}=\boldsymbol{\nabla} A \times \frac{\hat{\varphi}}{r \sin \theta}$

of the axisymmetric $\boldsymbol{B}$ in terms of the so-called flux function $A(r, \theta)$, which we impose to vanish on the $z$-axis. In particular, we have

$B=B_{\theta}=-\frac{1}{r} \frac{\partial A}{\partial r} \quad$ on $P$.

\subsection{Electric field}

The electric field $\boldsymbol{E}$ is purely electrostatic ( $\boldsymbol{B}$ is constant), and it has two components. The first one, $\boldsymbol{E}_{*}=-\boldsymbol{\nabla} \psi_{*}$, is created by the intrinsic bulk and surface distributions of charges in the pulsar, and its potential $\psi_{*}(r, \theta)$ is the solution tending to zero at infinity of the boundary value problem

$-\nabla^{2} \psi_{*}=0 \quad$ in $D$,

$\psi_{*}=\frac{\Omega_{*}}{c} A+\frac{Q_{s}}{r_{*}} \quad$ on $S$,

where $Q_{s}$ is a constant. A standard calculation shows that

$\psi_{*}(r, \pi / 2)=\frac{Q_{*}}{r}+\frac{m_{*} r_{*}^{2} \Omega_{*}}{3 c r^{3}} g(r)$,

where

$Q_{*}=\frac{2 \Omega_{*} m_{*}}{3 c}+Q_{s}$

is the net constant electric charge of the pulsar and $g(r)$ is a function depending on the flux distribution on $S$ (when $\boldsymbol{B}$ is dipolar, $g(r)=1$ and Eq. (6) reduces to the well-known result of Goldreich \& Julian 1969).

The second component of $\boldsymbol{E}, \boldsymbol{E}_{d}=-\boldsymbol{\nabla} \psi_{d}$, is generated by the disk charge density $\sigma$ and by the charge density $\sigma_{i}$ induced by the latter on the perfectly conducting surface $S$ of the pulsar. Its potential $\psi_{d}(r, \theta, \varphi, t)$ at time $t$ is the solution to the problem

$-\nabla^{2} \psi_{d}=4 \pi \sigma \delta(z) \quad$ in $D$

$\psi_{d}=\psi_{d}^{*}(t) \quad$ in $D_{*}$,

$\int_{S} \frac{\partial \psi_{d}}{\partial r} \mathrm{~d} s=0$

$\lim _{r \rightarrow \infty} \psi_{d}=0$.

Equation (8) is the standard Poisson equation in $D(\delta$ denotes the Dirac function), Eq. (9) is a consequence of the perfect conductivity of the outer layers of the star, which imposes indeed $\psi_{d}$ to be uniform in $D_{*}$ (note that $\psi_{d}^{*}(t)$ is not given a priori, its determination is part of the problem), Eq. (10) expresses the fact that the total charge induced on $S$ has to vanish (Gauss law is used here, with the integral over $S$ being computed on the outer side of that surface), and Eq. (11) is the usual asymptotic condition.

Note that a different decomposition of $\psi$ is made in Pétri et al. (2003), where the potential due to $\sigma_{i}$ is included in $\psi_{*}$. 


\subsection{Plasma motion}

Our next assumption fixes the way the disk particles move in the crossed electric field $\boldsymbol{E}=-\nabla \psi\left(\psi=\psi_{*}+\psi_{d}\right)$ and magnetic field $\boldsymbol{B}$. We adopt here the drift approximation, i.e., we take the plasma velocity to be given by

$\boldsymbol{v}=c \frac{\boldsymbol{E} \times \boldsymbol{B}}{B^{2}}=-c \frac{\boldsymbol{\nabla}_{s} \psi \times \hat{\boldsymbol{\theta}}}{B}$,

where $\boldsymbol{\nabla}_{s}=\hat{\boldsymbol{r}} \partial_{r}+(\hat{\boldsymbol{\varphi}} / r) \partial_{\varphi}$ is the gradient on $P$. To write the second equality, we have used the following facts: (i) the electric field is horizontal on $P$ owing to the mirror-symmetry of $\psi$ (the normal component of $\boldsymbol{E}$ suffers a discontinuity across $\Sigma-$ see Eq. (15) below -, but its values respectively on the upper and lower faces are opposite, and the electric field $E_{\theta}$ felt by the charges thus vanishes, which ensures equilibrium along $\boldsymbol{B}$ ); (ii) $\boldsymbol{\nabla}_{s} \psi$ is well defined on $P$ owing to the continuity of the tangential component of an electric field across a singular surface (Jackson 1975). Note that $\boldsymbol{v}$ has no component perpendicular to $\Sigma$ (the charges stay in the plane $P$ if they are in it initially, the model is consistent), and that

$v_{r}\left(r_{*}, \varphi, t\right)=\frac{c}{r_{*} B\left(r_{*}\right)} \frac{\partial \psi}{\partial \varphi}\left(r_{*}, \varphi, t\right)=0$

due to the axisymmetry of $\psi$ on $S$. Our assumption that no charges can be exchanged through $S$ is automatically satisfied.

The transport of the charges by the velocity $\boldsymbol{v}$ induces a change in the surface density $\sigma$, which evolves according to the continuity equation

$\frac{\partial \sigma}{\partial t}+\nabla_{s} \cdot(\sigma \boldsymbol{v})=0$

\subsection{Initial conditions}

The evolution of the disk can be determined by the following scheme. From the density $\sigma(r, \phi, t)$ at some time $t$, determine the potential $\psi_{d}(r, \theta, \phi, t)$ in $D$ by solving the problem (8)-(11). Next, compute $\boldsymbol{\nabla}_{s} \psi$ on $P$ and $\boldsymbol{v}$ by Eq. (12), and use the latter value in Eq. (14) to obtain $\sigma(r, \phi, t+\mathrm{d} t)$. Then return to step one. To initialize the computation, impose the condition $\sigma(r, \phi, t=$ $0)=\sigma_{0}(r, \phi)$.

As to the function $\sigma_{0}$, we will assume here that: (i) it is differentiable over $P$; (ii) $\sigma_{0}\left(r_{*}, \varphi\right)=0$, for otherwise the angular velocity of the plasma would diverge near the inner edge of the disk (see the end of the next section); (iii) $r^{3} \sigma_{0}=O(1)$ for large values of $r$ in the case where the disk is unbounded, this condition ensuring in particular the finiteness of the total disk charge $Q_{d}$, obviously conserved during the later evolution.

Note that: (i) in spite of the fact that the simulations quoted in the introduction show $\sigma$ to have the same sign as $Q_{*}$ everywhere, we have not required $\sigma_{0}$ to be of a single sign over $P$. This is just because considering the most general case does not lead to specific complications, and models may appear in which charges of both signs coexist in $P$; (ii) we have not excluded the possibility that the disk has an outer boundary located very far from the star or even extends to infinity, the latter situation being quite interesting from a theoretical point of view as it makes it easy to produce analytical explicit examples. Of course, this may lead to a physical problem as particles located at large distances are moving too fast $\left(E \sim r^{-2}\right.$ and $B \sim r^{-3}$, whence $\left.v_{\varphi} \sim r\right)$. However, this problem does not appear explicitly in the mathematics of the model, and it may be cared for to a large extent in the actual applications by choosing in any case $\sigma_{0}$ to be mostly concentrated not too far from the star, which keeps negligible the number of unphysical particles. Other physical constraints which have to be satisfied by $\sigma_{0}$ for the model to be valid are discussed in details in Pétri et al. (2002b), and are not repeated here.

\section{Some relations for the electrostatic potential and field}

We now derive a few relations satisfied by $\psi_{d}$ and $\boldsymbol{E}_{d}$. As already noted, the sources of these quantities are the charge densities $\sigma$ on $\Sigma$ and $\sigma_{i}$ on $S$ which are associated with the normal field discontinuities (Jackson 1975)
$\llbracket E_{d \theta} \rrbracket=2 E_{d \theta}^{+}=4 \pi \sigma$
on $\Sigma$,
$\llbracket E_{d r} \rrbracket=E_{d r}^{+}=4 \pi \sigma_{i}$
on $S$

(the + superscripts refer, respectively, to the lower side of $P$ and to the outer side of $S$ ). Of course,

$\int_{S} \sigma_{i} \mathrm{~d} s=0$,

which is just another form of Eq. (10). Note that Eq. (15) imposes $\sigma E_{\|}<0$ just above $\Sigma$ (with $E_{\|}$the component of $\boldsymbol{E}$ along $\boldsymbol{B})$, while in a true equilibrium electrosphere we would have $\mu E_{\|}>0$ just above the upper surface of the equatorial belt (approximated by $\Sigma$ in our model) where the volume charge density is $\mu$ (see Aly 2005, for a general proof of this statement). However, this should not cause too much trouble here, as the problems we are interested in are essentially dominated by the radial structure of the electric field (remember that we do not allow vertical distortions of $\Sigma$ ).

We now express explicitly the potential $\psi_{d}$ in $D$-i.e., the solution to Eqs. (8)-(11) - in terms of $\sigma$. This can be done by using the method of images, which leads to

$$
\begin{aligned}
\psi_{d}(\boldsymbol{r}) & =\int_{P} G\left(r, r^{\prime}, \varphi-\varphi^{\prime}\right) \sigma\left(\boldsymbol{r}^{\prime}\right) \mathrm{d} s^{\prime} \\
& =\int_{P}\left[\frac{1}{\left|\boldsymbol{r}-\boldsymbol{r}^{\prime}\right|}-\frac{r_{*} / r^{\prime}}{\left|\boldsymbol{r}-\left(r_{*} / r^{\prime}\right)^{2} \boldsymbol{r}^{\prime}\right|}+\frac{r_{*} / r^{\prime}}{r}\right] \sigma\left(\boldsymbol{r}^{\prime}\right) \mathrm{d} s^{\prime}
\end{aligned}
$$

(Jackson 1975, p. 58). The Green function $G$ of the problem (given by the bracket in the last member) has three terms: (i) the first gives the potential of a unit charge located at $\boldsymbol{r}^{\prime}$; (ii) the second represents the potential of an image charge $-r_{*} / r^{\prime}$ located at $\left(r_{*} / r^{\prime}\right)^{2} \boldsymbol{r}^{\prime}$ and it combines with the first to give a zero potential on $S$; (iii) the third is the potential of a central charge $r_{*} / r^{\prime}$ which makes the total image charge equal to zero, and creates a uniform potential on $S$. Note that the integral in Eq. (18) converges for our disk with a finite charge.

The value of $\psi_{d}$ on $S$ (and then in $D_{*}$ ) is obtained by choosing $r=r_{*}$ in Eq. (18). From the interpretation above of $G$, the 
first two contributions to $\psi_{d}^{*}$ compensate each other, and we are left with

$\psi_{d}^{*}=\int_{P} \frac{\sigma}{r} \mathrm{~d} s$

Note that the latter expression can also be obtained by taking $\boldsymbol{r}=0$ in the standard relation

$\psi_{d}(\boldsymbol{r})=\int_{P} \frac{\sigma\left(\boldsymbol{r}^{\prime}\right)}{\left|\boldsymbol{r}-\boldsymbol{r}^{\prime}\right|} \mathrm{d} s^{\prime}+\int_{S} \frac{\sigma_{i}\left(\boldsymbol{r}^{\prime}\right)}{\left|\boldsymbol{r}-\boldsymbol{r}^{\prime}\right|} \mathrm{d} s^{\prime}$

valid in the whole space and by using Eq. (17).

From Eq. (18) we can obtain an explicit formula for $\boldsymbol{\nabla}_{s} \psi_{d}$ on $P$ and thus one for $\boldsymbol{v}$ by Eq. (12), and using the latter in the continuity Eq. (14) leads to an integro-differential equation satisfied by the only variable $\sigma$. Once an initial condition has been fixed, we obtain that way an alternative formulation of the evolution problem.

Actually, some care is needed for the computation of $\boldsymbol{E}_{d}=$ $-\nabla_{s} \psi_{d}$, as the gradient of $G$ contains two terms which are nonintegrable in a neighborhood of $\boldsymbol{r}$ when the latter is taken, respectively, on $P$ and on the star equator $\partial P$. The right procedure is to first compute the gradient of $\psi_{d}$ in $D \backslash P$, and next to take the limit $\theta \rightarrow \pi / 2$. One then gets an expression in which an integral occurs which has to be understood as a principal value. Also, if $\sigma$ either suffered a jump along some curve $C$ or did not vanish along $\partial P$, the singular character of the kernel in the integral giving $\boldsymbol{E}_{d}$ would make that field diverging according to $E_{d} \propto \ln \rho$, with $\rho$ the distance to $C$ or $\partial P$. Such a behaviour keeps the electrostatic energy finite, but implies infinite angular velocities, and it is to prevent this type of unpleasant divergence that we have imposed assumptions (i) and (ii) in Sect. 2.4.

\section{Hamiltonian forms of the equations}

\subsection{An alternative form of the continuity equation}

Noticing that

$B \boldsymbol{v}=-c \boldsymbol{\nabla}_{s} \psi \times \hat{\boldsymbol{\theta}}=-c \boldsymbol{\nabla}_{s} \times(\psi \hat{\boldsymbol{\theta}})$,

we get

$\boldsymbol{\nabla}_{s} \cdot(B \boldsymbol{v})=0$,

and we can rewrite Eq. (14) in the form

$$
\frac{\partial \sigma}{\partial t}+B \boldsymbol{v} \cdot \boldsymbol{\nabla}_{s} \frac{\sigma}{B}=0
$$

Therefore, the amount of electric charge per unit of magnetic flux, $\eta=\sigma / B$, does satisfy

$\frac{\mathrm{d} \eta}{\mathrm{d} t}=\frac{\partial \eta}{\partial t}+\boldsymbol{v} \cdot \boldsymbol{\nabla}_{s} \eta=0$

$\eta$ keeps a constant value when we follow the motion of a plasma element.

\subsection{Equations in canonical Hamiltonian form}

A most convenient form of Eq. (24) can be obtained by using $a=A / c$ as the radial variable, which is possible because $A(r, \pi / 2)$ is a monotonically decreasing function of $r$ (to see this, just use in Eq. (3) the condition $B_{\theta}>0$ we have imposed on $P)$. In coordinates $(\varphi, a)$, the equations are defined in the rectangular domain $\Pi=\left\{0 \leq \varphi \leq 2 \pi ; 0<a<a_{m}\right\}$, with the sides $\Lambda_{0}=\{a=0\}$ and $\Lambda=\left\{a=a_{m}=A\left(r_{*}, \pi / 2\right) / c\right\}$ corresponding, respectively, to the circle at infinity in $P$ and to the equator of the star, and the sides $\{\varphi=0\}$ and $\{\varphi=2 \pi\}$ being identified. Then all the functions $f(\varphi, a)$ we shall have to consider will be understood to satisfy $f(0, a)=f(2 \pi, a)$. For such functions $f$ and $g$ we set

$[f, g]=\frac{\partial(f, g)}{\partial(\varphi, a)}=\frac{\partial f}{\partial \varphi} \frac{\partial g}{\partial a}-\frac{\partial f}{\partial a} \frac{\partial g}{\partial \varphi}$.

As is easily checked, $[.,$.$] is antisymmetric ([f, g]=-[g, f])$, is a derivation $([f, g h]=[f, g] h+[f, h] g)$ and satisfies Jacobi's identity $([[f, g], h]+[[h, f], g]+[[g, h], f]=0)$. Using the relation $\mathrm{d} a=-r B \mathrm{~d} r / c$ (see Eq. (3)), we thus get from Eq. (12)

$\frac{\mathrm{d} \varphi}{\mathrm{d} t}=[\varphi, \psi]=\frac{\partial \psi}{\partial a}$
$\frac{\mathrm{d} a}{\mathrm{~d} t}=[a, \psi]=-\frac{\partial \psi}{\partial \varphi}$

while Eq. (24) takes the form

$\frac{\partial \eta}{\partial t}+[\eta, \psi]=0$

Then $\eta$ is transported in $\Pi$ at the divergenceless velocity $\left(\partial_{a} \psi,-\partial_{\varphi} \psi\right)$. The flow in $\Pi$ turns out to be incompressible, contrarily to the physical flow in $P$ which is compressible owing to the nonuniformity of $B$.

Equations (26) and (27) appear to have the form of the canonical equations determining the evolution of a one degree of freedom Hamiltonian mechanical system, with $\varphi$ and $a$ being a pair of canonically conjugated variables defining the phase space $\Pi,[.,$.$] being the associated Poisson bracket and \psi(\varphi, a)$ being the Hamiltonian. Moreover, Eq. (28) has the form of the Liouville equation governing the transport in $\Pi$ of the density distribution $\eta$.

However, the analogy is not complete. For a usual mechanical system, the Hamiltonian is a given function on $\Pi$, while it has to be determined here from the density $\eta$ by using the relation

$$
\begin{aligned}
\psi(\varphi, a) & =\psi_{*}(a)+\psi_{d}(\varphi, a) \\
& =\psi_{*}(a)+\int_{\Pi} G\left(\varphi-\varphi^{\prime}, a, a^{\prime}\right) \eta\left(\varphi^{\prime}, a^{\prime}\right) \mathrm{d} \tau^{\prime},
\end{aligned}
$$

where $G\left(\varphi-\varphi^{\prime}, a, a^{\prime}\right)$ is the Green function defined by Eq. (18) and $\mathrm{d} \tau=\mathrm{d} \varphi \mathrm{d} a=B \mathrm{~d} s$ is the surface element in $\Pi$. Fortunately, a fully Hamiltonian description, albeit one of the non-canonical type, can be established as in most ideal fluid theories (see, e.g., Morrison 1998). This is shown in Appendix A, whose results are summarized in the next subsection. As indicated in the introduction, there is no problem in skipping this short part of the paper. 


\subsection{Non-canonical Hamiltonian structure of the equations}

To set up an Hamiltonian framework for our disk (which is a continuous system, with an infinite number of degrees of freedom), we deal with arbitrary functionals $\mathcal{F}$ acting on the functions $\eta(\varphi, a)$ defined on $\Pi$ rather than with the actual density $\eta$ itself - a functional $\mathcal{F}$ being just a mapping associating to $\eta$ a real number $\mathcal{F}[\eta]$. The two basic ingredients of the formalism are the Lie-Poisson bracket $\{\mathcal{F}, \mathcal{G}\}$ of two functionals $\mathcal{F}$ and $\mathcal{G}$, defined by

$\{\mathcal{F}, \mathcal{G}\}[\eta]=\int_{\Pi}\left[\frac{\delta \mathcal{F}}{\delta \eta}, \frac{\delta \mathcal{G}}{\delta \eta}\right] \eta \mathrm{d} \tau$

$(\delta \mathcal{F} / \delta \eta$ denotes the so-called functional derivative of $\mathcal{F})$, and the Hamiltonian functional $\mathcal{H}$, defined by

$\mathcal{H}[\eta]=\frac{1}{2} \int_{\Pi \times \Pi} G \eta \eta^{\prime} \mathrm{d} \tau \mathrm{d} \tau^{\prime}+\int_{\Pi} \psi_{*} \eta \mathrm{d} \tau$.

Thus a solution $\eta(t)$ to our problem is determined by the equation

$\frac{\mathrm{d}}{\mathrm{d} t} \mathcal{F}[\eta(t)]=\{\mathcal{F}, \mathcal{H}\}[\eta(t)]$,

where $\mathcal{F}$ is an arbitrary functional not depending explicitly on time.

\subsection{A property of $[.,$.}

To conclude this section, we give a useful property of the bracket [., .]. Let $f, g$ and $h$ be three functions on $\Pi$. By using the antisymmetry of [., .], its property of being a derivation and its explicit definition, we get

$$
\begin{aligned}
\int_{\Pi}[f, g] h \mathrm{~d} \tau= & \int_{\Pi}[h, f] g \mathrm{~d} \tau+\int_{\Pi}[f, g h] \mathrm{d} \tau=\int_{\Pi}[h, f] g \mathrm{~d} \tau \\
& +\int_{\Pi}\left[\frac{\partial}{\partial \varphi}\left(f \frac{\partial(g h)}{\partial a}\right)-\frac{\partial}{\partial a}\left(f \frac{\partial(g h)}{\partial \varphi}\right)\right] \mathrm{d} \tau .
\end{aligned}
$$

In the last member, the first term in the second integral disappears because of the $\varphi$-periodicity of the functions, and after integration of the second one with respect to $a$ we get

$$
\begin{aligned}
\int_{\Pi}[f, g] h \mathrm{~d} \tau= & \int_{\Pi}[h, f] g \mathrm{~d} \tau+\int_{\Lambda_{0}} f \frac{\partial(g h)}{\partial \varphi} \mathrm{d} \varphi \\
& -\int_{\Lambda} f \frac{\partial(g h)}{\partial \varphi} \mathrm{d} \varphi .
\end{aligned}
$$

In particular, we obtain

$\int_{\Pi}[f, g] h \mathrm{~d} \tau=\int_{\Pi}[h, f] g \mathrm{~d} \tau$

when either $f$ or $g h$ does not depend on $\varphi$ on $\Lambda_{0}$ and $\Lambda$.

\section{Conservation laws}

In this section, $(\eta(t), \psi(t))$ denotes an arbitrary solution to the equations of the disk.

\subsection{A family of Lagrangian invariants}

We first note that the conservation of $\eta$ along the trajectory of a plasma element implies the conservation law

$\|\eta(t)\|=\sup _{\Pi}|\eta(t)|=\sup _{\Pi}|\eta(0)|=\left\|\eta_{0}\right\|<\infty$

(when $\Sigma$ is unbounded, the statement $\left\|\eta_{0}\right\|<\infty$ is a consequence of our assumption $\sigma_{0} r^{3}=O(1)$ for $r$ large).

Consider now some arbitrary function $f(s)$ which is bounded for $|s| \in\left[0,\left\|\eta_{0}\right\|\right]$, and introduce the well defined quantity

$F(t)=\int_{\Pi} f(\eta(t)) \mathrm{d} \tau=\frac{1}{c} \int_{P} f(\eta(t)) B \mathrm{~d} s$.

Using once more the conservation of $\eta$ when one follows an element of plasma, as well as the conservation of $\mathrm{d} \tau$, we can immediately state that $F$ is conserved, i.e.,

$F(t)=F(0)$.

Alternatively, this conclusion can be obtained by an explicit calculation in Eulerian variables. Using Eqs. (28) and (35) (with $h=1$ and $f=\psi$ independent of $\varphi$ on $\Lambda_{0}$ and $\Lambda$ ), we obtain indeed

$\frac{\mathrm{d} F}{\mathrm{~d} t}=-\int_{\Pi} f^{\prime}(\eta)[\eta, \psi] \mathrm{d} \tau=\int_{\Pi}[\psi, f(\eta)] \mathrm{d} \tau=0$.

(If $f$ has discontinuities, the derivation still works by allowing the presence of $\delta$-type singularities in $f^{\prime}$ ).

As an example, let us choose for $f$ the parametrized function $f_{\delta}(s)=\Theta(|s|-\delta)$, with $\Theta$ the standard Heavyside step function and $\delta \in[0, \infty[$. This leads to the conservation law

$\mathcal{A}_{\eta(t)}(\delta)=\mathcal{A}_{\eta_{0}}(\delta)$,

where

$\mathcal{A}_{\eta}(\delta)=\int_{\Pi} \Theta[|\eta|-\delta] \mathrm{d} \tau=\int_{\{|\eta| \geq \delta\}} \mathrm{d} \tau$

is the area of that part of $\Pi$ on which $|\eta| \geq \delta$.

Also, taking $f(s)=s$ (resp., $f(s)=|s|$ ) leads to the expected conservation of the total charge $Q_{d}$ of the disk (resp., the total unsigned charge $Q_{d}^{u}=\int_{P}|\sigma| \mathrm{d} s$ ).

\subsection{Angular momentum conservation}

We next introduce the quantity

$J(t)=\int_{\Pi} a \eta(t) \mathrm{d} \tau=\frac{1}{c} \int_{P} A \sigma(t) \mathrm{d} s$,

which is well defined for our disk with a finite charge (we have $\left.|J(t)| \leq a_{m} Q_{d}^{u}<\infty\right)$. To interpret it, we recall that $a=A / c$ is the momentum conjugate to $\varphi$ in the Hamiltonian formulation, and then $J \hat{z}$ appears to be the moment with respect to $O$ of the momenta of all the disk particles. (More generally, it is well known that a particle of mass $m$ and charge $q$ located in a magnetic field can be assigned a generalized momentum $m \boldsymbol{v}+q \boldsymbol{A} / c$, where $\boldsymbol{A}$ is a potential vector for $\boldsymbol{B}$. Here, we choose 
$\boldsymbol{A}=(A / r \sin \theta) \hat{\boldsymbol{\varphi}}$ and set $m=0$ because of our drift approximation).

Let us compute the time-derivative of $J$. With the help of Eqs. (28) and (35) and the definition (25) of the Poisson bracket, we obtain

$\frac{\mathrm{d} J}{\mathrm{~d} t}=\int_{\Pi} a[\psi, \eta] \mathrm{d} \tau=\int_{\Pi}[a, \psi] \eta \mathrm{d} \tau=-\int_{\Pi} \frac{\partial \psi_{d}}{\partial \varphi} \eta \mathrm{d} \tau$.

On the other hand, by using Eq. (29) and the fact that $G=$ $G\left(\varphi-\varphi^{\prime}, a, a^{\prime}\right)$ we get

$$
\begin{aligned}
-\int_{\Pi} \frac{\partial \psi_{d}}{\partial \varphi} \eta \mathrm{d} \tau & =-\int_{\Pi \times \Pi} \frac{\partial G}{\partial \varphi} \eta \eta^{\prime} \mathrm{d} \tau \mathrm{d} \tau^{\prime} \\
& =\int_{\Pi \times \Pi} \frac{\partial G}{\partial \varphi^{\prime}} \eta \eta^{\prime} \mathrm{d} \tau \mathrm{d} \tau^{\prime}=\int_{\Pi} \frac{\partial \psi_{d}^{\prime}}{\partial \varphi^{\prime}} \eta^{\prime} \mathrm{d} \tau^{\prime}
\end{aligned}
$$

As the last member is the reverse of the first one, both vanish, and we can eventually conclude that

$\frac{\mathrm{d} J}{\mathrm{~d} t}=0$

Therefore $J$ - to which we shall refer as the angular momentum - is conserved:

$J(t)=J(0)$.

It is worth noticing that Eqs. (46) and (19) imply

$\psi_{d}^{*}(t)=\int_{P} \frac{\sigma}{r} \mathrm{~d} s=\int_{P} \frac{\sigma_{0}}{r} \mathrm{~d} s=\psi_{d}^{*}(0)$

when the pulsar magnetic field $\boldsymbol{B}$ is taken to be dipolar, i.e., $A=m_{*} r^{-1}$ on $P$. The uniform value of $\psi_{d}$ in $D_{*}$ is also constant in time!

Finally, we consider the electromagnetic angular momentum

$L_{z}=\hat{z} \cdot \int_{D} \boldsymbol{r} \times \frac{\boldsymbol{E} \times \boldsymbol{B}}{4 \pi c} \mathrm{~d} v=\frac{1}{4 \pi c} \int_{D} \boldsymbol{\nabla} A \cdot \nabla \psi \mathrm{d} v$

introduced in Pétri et al. (2003) (see their Eqs. (A.5) and (A.7)). By using Eq. (8) and carrying out an integration by parts, we get the relation

$J=L_{z}+\frac{1}{4 \pi c} \int_{S} A \frac{\partial \psi}{\partial r} \mathrm{~d} s=L_{z}+L_{s}$.

Owing to the assumption $\boldsymbol{\nabla} \cdot\left(\partial_{t} \boldsymbol{E}+4 \pi \boldsymbol{j}\right)=0(\boldsymbol{j}=$ current density) of the electrostatic model (here, our Eq. (14)), we have $\partial_{t} \boldsymbol{E}+4 \pi \boldsymbol{j}=c \boldsymbol{\nabla} \times \boldsymbol{b}$, with $\boldsymbol{b} \neq 0$ in general, and it is easily shown that the existence of a component $b_{\varphi} \neq 0$ on $S$ makes $\mathrm{d}_{t} L_{s}$ non strictly vanishing. However, consistently with the neglect of $\boldsymbol{b}$ in the model, the a posteriori computed quantity $b / B$ is expected on general grounds (J. Heyvaerts 2004, private communication) to be at most of the order of $(v / c)^{2}$, with $v$ the typical velocity of the disk particles. Therefore, the value obtained for $\mathrm{d}_{t} L_{z}=-\mathrm{d}_{t} L_{s}$ is quite small, and $L_{z}$ can be taken to be conserved at the order of approximation defining the model, as assumed in Pétri et al. (2003).

\subsection{Electrostatic energy conservation}

Finally, we consider the electrostatic energy associated with the introduction of the disk in the environment of the star (with our assumptions, the energy of the pulsar intrinsic charges is constant and of no interest here). This quantity has two parts. The first results from the self-interaction of the charges creating $\psi_{d}$, i.e., the charges in the disk and those they induce on $S$, and is given by

$W_{1}=\frac{1}{2} \int_{P} \sigma \psi_{d} \mathrm{~d} s+\frac{1}{2} \int_{S} \sigma_{i} \psi_{d} \mathrm{~d} s=\frac{1}{2} \int_{P} \sigma \psi_{d} \mathrm{~d} s$,

the second equality being an immediate consequence of Eqs. (9) and (17). Alternatively, we can write with the help of Eq. (29)

$W_{1}=\frac{1}{2} \int_{\Pi \times \Pi} G \eta \eta^{\prime} \mathrm{d} \tau \mathrm{d} \tau^{\prime}$,

and, by using Eq. (8), the Green first identity and the boundary conditions (9)-(10) on $S$,

$W_{1}=\int_{D} \frac{\left|\nabla \psi_{d}\right|^{2}}{8 \pi} \mathrm{d} v$.

The second part of the energy is related to the interaction of these same charges with the constant potential $\psi_{*}$. It is given by

$W_{2}=W_{d *}+W_{i *}=\int_{P} \sigma \psi_{*} \mathrm{~d} s+\int_{S} \sigma_{i} \psi_{*} \mathrm{~d} s$,

and it can be expressed as

$W_{2}=\psi_{d}^{*} Q_{*}$.

Indeed by using successively Eqs. (4) and (8), the Green second identity in $D$, and Eqs. (9) and (16), we obtain

$$
\begin{aligned}
4 \pi \int_{P} \sigma \psi_{*} \mathrm{~d} s & =\int_{D}\left(\psi_{d} \nabla^{2} \psi_{*}-\psi_{*} \nabla^{2} \psi_{d}\right) \mathrm{d} v \\
& =\int_{S}\left(-\psi_{d} \frac{\partial \psi_{*}}{\partial r}+\psi_{*} \frac{\partial \psi_{d}}{\partial r}\right) \mathrm{d} s \\
& =-\psi_{d}^{*} \int_{S} \frac{\partial \psi_{*}}{\partial r} \mathrm{~d} s-4 \pi \int_{S} \sigma_{i} \psi_{*} \mathrm{~d} s
\end{aligned}
$$

from which Eq. (54) follows when the Gauss relation

$-\int_{S} \frac{\partial \psi_{*}}{\partial r} \mathrm{~d} s=4 \pi Q_{*}$

is taken into account.

Let us now consider the quantity

$W=W_{1}+W_{d *}$

and compute its derivative. Using the expression (51) for $W_{1}$ and Eq. (35), we obtain

$$
\begin{aligned}
\frac{\mathrm{d} W}{\mathrm{~d} t} & =\int_{\Pi}\left(\psi_{d}+\psi_{*}\right) \frac{\partial \eta}{\partial t} \mathrm{~d} \tau \\
& =\int_{\Pi} \psi[\psi, \eta] \mathrm{d} \tau=\int_{\Pi}[\psi, \psi] \eta \mathrm{d} \tau=0
\end{aligned}
$$

and therefore $W$ is conserved, i.e.,

$W(t)=W(0)$. 
As for the true total electrostatic energy $W_{e}=W_{1}+W_{2}=$ $W+W_{i *}$, we obtain by using Eqs. (53), (5) and (58)

$\frac{\mathrm{d} W_{e}}{\mathrm{~d} t}=\frac{\mathrm{d}}{\mathrm{d} t} \int_{S} \sigma_{i} \psi_{*} \mathrm{~d} s=-\frac{\Omega_{*}}{4 \pi c} \frac{\mathrm{d}}{\mathrm{d} t} \int_{S} \frac{\partial \psi}{\partial r} A \mathrm{~d} s$,

whence, with the help of Eqs. (49) and (45),

$\frac{\mathrm{d} W_{e}}{\mathrm{~d} t}=\Omega_{*} \frac{\mathrm{d} L_{z}}{\mathrm{~d} t}$

Note that this relation between the variation of the electromagnetic energy and the (small) variation of the electromagnetic angular momentum is coherent with the fact that these quantities can change only because of exchanges with the rigidly rotating star.

\section{Confinement theorems}

\subsection{General statements}

During the evolution of the disk from some initial configuration, some of the particles initially located near the star may be expelled to large distances. The aim of this section is to derive some a priori bounds on the amount of charge which can be lost that way by the system. Of course, particle escape is associated to a positive value of the radial velocity in some part of $\Sigma$, and we start with two general remarks on that quantity.

With an overline denoting a $\varphi$-average, we have

$\overline{v_{r}}(r)=\frac{c}{B(r)} \int_{0}^{2 \pi} \frac{\partial \psi}{\partial \varphi}(r, \varphi) \frac{\mathrm{d} \varphi}{2 \pi}=0$,

and the flux of charge (electric current) through a circle of radius $r$ is given by

$I(r)=2 \pi r \overline{\sigma v_{r}}=2 \pi r \overline{(\sigma-\bar{\sigma}) v_{r}}=\int_{0}^{2 \pi}(\sigma-\bar{\sigma}) v_{r} r \mathrm{~d} \varphi$.

Therefore: (i) there is no radius $r$ at which the particles are all moving towards larger (or smaller) radii; if $v_{r}(r, \varphi)>0$ in some range of $\varphi$, there must be a complementary range in which $v_{r}(r, \varphi)<0$; (ii) the current $I(r)$ can be described as the transport of the fluctuation $\sigma-\bar{\sigma}$ of the charge density, which in many cases may be much smaller (in absolute value) than $\sigma$.

We now introduce the amount of unsigned charge

$Q_{d}^{u}(r, t)=\int_{P_{r}}|\sigma(t)| \mathrm{d} s=\int_{\{c a \leq A(r)\}}|\eta(t)| \mathrm{d} \tau$

contained in that part $P_{r}$ of $P$ located beyond radius $r$, and show that some of the conservation laws previously derived can be used for establishing confinement theorems, that is to say inequalities of the form

$\frac{Q_{d}^{u}(r, t)}{Q_{d}^{u}} \leq h(r)$

with $h(r) \leq 1$ a function (independent of time) fully determined by the bounded initial function $\eta_{0}$.

\subsection{First theorem}

By angular momentum conservation, we have

$J(0)=\int_{\{c a \leq A(r)\}} \eta(t) a \mathrm{~d} \tau+\int_{\{A(r)<c a\}} \eta(t) a \mathrm{~d} \tau$

at any time $t$. Then

$$
\begin{aligned}
|J(0)| & \leq \frac{A(r)}{c} \int_{\{c a \leq A(r)\}}|\eta(t)| \mathrm{d} \tau+a_{m} \int_{\{A(r)<c a\}}|\eta(t)| \mathrm{d} \tau \\
& =-\left(a_{m}-\frac{A(r)}{c}\right) Q_{d}^{u}(r, t)+a_{m} Q_{d}^{u}
\end{aligned}
$$

and

$\frac{Q_{d}^{u}(r, t)}{Q_{d}^{u}} \leq \frac{1-|J(0)| /\left(a_{m} Q_{d}^{u}\right)}{1-A(r) /\left(c a_{m}\right)}$.

In particular, we get

$\lim _{r \rightarrow \infty} \frac{Q_{d}^{u}(r, t)}{Q_{d}^{u}} \leq 1-\frac{|J(0)|}{a_{m} Q_{d}^{u}}$,

which implies that not all the charges can escape to infinity. When the sign of $\eta$ is uniform over $\Sigma$ and the field is dipolar (i.e., $\left.A(r) /\left(c a_{m}\right)=r_{*} / r\right)$, we essentially recover the result in Pétri et al. (2003).

\subsection{Second theorem}

Let us introduce the set $\mathcal{K}\left[\eta_{0}\right]$ of all the functions $\eta(\varphi, a)$ which satisfy the constraint $\mathcal{A}_{\eta}(\delta)=\mathcal{A}_{\eta_{0}}(\delta), \delta \geq 0 . \mathcal{K}\left[\eta_{0}\right]$ contains in particular a unique non-negative function $\widetilde{\eta_{0}}$ which is independent of $\varphi$ and is non-increasing with respect to $a$, and it is quite obvious that

$$
\begin{aligned}
\int_{\{c a<A(r)\}}|\eta| \mathrm{d} \tau & \leq \int_{\{c a<A(r)\}} \widetilde{\eta_{0}} \mathrm{~d} \tau \\
& =2 \pi \int_{0}^{A(r) / c} \widetilde{\eta_{0}}(a) \mathrm{d} a
\end{aligned}
$$

for any $\eta \in \mathcal{K}\left[\eta_{0}\right]$ and any $r>r_{*}$. Next we note that $\eta(t) \in$ $\mathcal{K}\left[\eta_{0}\right]$ at any time $t$ as a consequence of the area conservation law (40), and Eq. (70) can thus be applied to it, which leads to our second confinement theorem

$\frac{Q_{d}^{u}(r, t)}{Q_{d}^{u}} \leq \frac{2 \pi}{Q_{d}^{u}} \int_{0}^{A(r) / c} \widetilde{\eta_{0}}(a) \mathrm{d} a$.

A weaker but more convenient form of this inequality can be obtained by noticing that

$\left\|\widetilde{\eta_{0}}\right\|=\sup _{\Pi} \widetilde{\eta_{0}}=\sup _{\Pi}\left|\eta_{0}\right|=\left\|\eta_{0}\right\|$

whence

$\frac{Q_{d}^{u}(r, t)}{Q_{d}^{u}} \leq \frac{2 \pi\left\|\eta_{0}\right\| A(r)}{c Q_{d}^{u}} \leq \frac{2 \pi\left\|\eta_{0}\right\| m}{c r Q_{d}^{u}}$.

It thus appears that the fraction of unsigned charge located beyond the radius $r$ is bounded at any time $t$ by a timeindependent function decreasing to zero as $r^{-1}$ for large values of $r$. Clearly, this result precludes any amount of charge 
reaching infinity, and is then much more stringent than the one derived above. We also note that the additional introduction of angular momentum conservation may lead to a slight improvement of Eq. (71), but at the expense of a somewhat cumbersome discussion that we shall avoid presenting here.

\section{Steady states and their stability}

\subsection{Steady states}

We define here a steady state $\left(\eta^{0}, \psi^{0}\right)$ to be a solution of our problem that is independent of time in some frame uniformly rotating around $\hat{z}$ at constant angular velocity $\Omega_{r}$. Allowing the possibility of having $\Omega_{r} \neq 0$ is important, firstly because rotating coherent nonaxisymmetric structures may possibly form in a pulsar magnetosphere as they do in laboratory nonneutral plasmas (e.g., Davidson \& Lund 1991), and secondly because this provides a quite useful trick for studying the stability of axisymmetric systems which are steady in any rotating frame.

For a steady state with a pattern rotating at $\Omega_{r}$, we have

$\frac{\partial \eta^{0}}{\partial t}=-\Omega_{r} \frac{\partial \eta^{0}}{\partial \varphi}=-\left[\eta^{0}, \Omega_{r} a\right]$

and by plugging this relation into the continuity Eq. (24) we get

$\frac{\partial \eta^{0}}{\partial t}+\left[\eta^{0}, \psi^{0}\right]=\left[\eta^{0}, \psi^{0}-\Omega_{r} a\right]=0$.

Introducing the potential in the rotating frame,

$\chi^{0}=\psi^{0}-\Omega_{r} a=\psi^{0}-\frac{\Omega_{r} A}{c}$,

we thus have

$\left[\eta^{0}, \chi^{0}\right]=0$,

which implies that $\eta^{0}$ is a function (possibly multivalued) of $\chi^{0}$, i.e.,

$\eta^{0}=h\left(\chi^{0}\right)$

Reciprocally, the system is in a steady state if a relation of the form (78) holds for $\eta^{0}$.

The potential $\chi^{0}$ in $\Pi$ has to satisfy

$\chi^{0}(\varphi, a)+\Omega_{r} a=\int_{\Pi} G\left(\varphi-\varphi^{\prime}, a, a^{\prime}\right) h\left(\chi^{0}\left(\varphi^{\prime}, a^{\prime}\right)\right) \mathrm{d} \tau^{\prime}$.

A steady state can thus be constructed by fixing $h$ and $\Omega_{r}$, and by solving the latter integral equation. For a given $h$, it is likely that non-axisymmetric solutions cannot exist for arbitrary values of $\Omega_{r}$, and a priori criteria (of the type of the one derived in Aly 1994, for laboratory plasmas) would certainly be useful to guide the search for them.

\subsection{A nonlinear stability theorem}

We now apply Arnold's general method (Holm et al. 1985) to derive a sufficient criterion of stability for a given rotating steady state. If we impose on the latter some perturbation at time $t=0$, the density $\eta$ switches from the steady value $\eta^{0}\left(\chi^{0}\right)$ to some value $\eta_{0}$, from which the system suffers for $t>0$ an evolution described by $\eta(t)$. Owing to the conservation laws derived in Sect. 5, the quantity $G=W-\Omega_{r} J+F$ is conserved for any functional $F$ of the form (37). Therefore

$\Delta G(t)=G(t)-G^{0}=G(0)-G^{0}=\Delta G(0)$,

where $G^{0}=G\left[\eta^{0}\right]$. If we set

$\eta(t)=\eta^{0}+\delta \eta(t)$

and introduce expressions (57) for $W$ and (42) for $J$, we obtain

$$
\begin{aligned}
\Delta G(0)= & \frac{1}{2} \int_{\Pi \times \Pi} G \delta \eta \delta \eta^{\prime} \mathrm{d} \tau \mathrm{d} \tau^{\prime}+\int_{\Pi}\left[\left(\chi^{0}+f^{\prime}\left(\eta^{0}\right)\right) \delta \eta\right. \\
& \left.+f\left(\eta^{0}+\delta \eta\right)-f\left(\eta^{0}\right)-f^{\prime}\left(\eta^{0}\right) \delta \eta\right] \mathrm{d} \tau .
\end{aligned}
$$

Finally, choosing the still arbitrary function $f$ such that

$f^{\prime}\left[\eta^{0}\left(\chi^{0}\right)\right]=-\chi^{0}$

- which is possible if $\eta^{0}\left(\chi_{0}\right)$ is a monotonic function - and applying the mean-value theorem leads to

$W_{1}[\delta \eta(t)]+\int_{\Pi} f^{\prime \prime}\left(\eta_{1}\right)(\delta \eta)^{2} \mathrm{~d} \tau=\Delta G(0)$,

where $\eta_{1}(\varphi, a, t)$ is such that $\left.\eta_{1} \in\right] \eta^{0}, \eta[$ and we have set

$W_{1}[\delta \eta(t)]=\frac{1}{2} \int_{\Pi \times \Pi} G \delta \eta \delta \eta^{\prime} \mathrm{d} \tau \mathrm{d} \tau^{\prime}=\int_{D} \frac{\left|\nabla \delta \psi_{d}\right|^{2}}{8 \pi} \mathrm{d} \nu$.

The second equality, where $\delta \psi_{d}(t)=\psi(t)-\psi^{0}$, results from Eq. (52) and it implies that $W_{1}[\delta \eta]$ is positive definite.

Let us now assume that $\eta^{0}$ satisfies

$-\frac{1}{k} \leq \frac{\mathrm{d} \eta^{0}}{\mathrm{~d} \chi^{0}}=-\frac{1}{f^{\prime \prime}\left(\eta^{0}\right)} \leq-\frac{1}{K}<0$,

with $k>0$ and $K>0$. Setting $\|\delta \eta(0)\|=\sup _{\Pi}|\delta \eta(0)|$, we thus get from Eqs. (84) and (86)

$W_{1}[\delta \eta(t)]+k \int_{\Pi}|\delta \eta(t)|^{2} \mathrm{~d} \tau \leq \kappa\|\delta \eta(0)\|^{2}$,

where $\kappa=W_{1}[1]+2 \pi a_{m} K$. Clearly, the latter relation shows that the amplitude of the perturbation at any time $t$ - measured by the left-hand side - can be made as small as we want by choosing the amplitude of the initial perturbation - measured by the right-hand side - small enough. This is just the definition of Liapunov nonlinear stability (relative to our specific choice of norms). Note that the weaker condition $\mathrm{d} \eta^{0} / \mathrm{d} \chi^{0}<0$ would allow us to conclude only at some form of linear stability of the system (Holm et al. 1985).

\subsection{The case of an axisymmetric steady state}

An axisymmetric steady state $\left(\eta^{0}(a), \psi^{0}(a)\right)$ can be constructed by fixing arbitrarily the density $\eta^{0}(a)$. Indeed, the associated potential $\psi^{0}$ in $\Pi$ is a function of $a$ only, and Eq. (78) is trivially satisfied for any value of $\Omega_{r}$ (steadiness holds in any uniformly rotating frame). $\Omega_{r}$ has no intrinsic meaning here and can be considered as a parameter whose value can be choosen at our 
convenience. We note that the plasma rotates at the angular velocity (see Eq. (26))

$\Omega^{0}(a)=\frac{\mathrm{d} \psi^{0}}{\mathrm{~d} a}(a)$,

and the derivative of $\chi^{0}(a)$ can thus be written

$\frac{\mathrm{d} \chi^{0}}{\mathrm{~d} a}(a)=\Omega^{0}(a)-\Omega_{r}$.

Let us then assume that $-K_{1} \leq \mathrm{d} \eta^{0} / \mathrm{d} a \leq-k_{1}$ for $0 \leq a \leq a_{m}$ and some $k_{1}>0$ and $K_{1}>0$ (this implies that we have an unbounded disk for which $\eta^{0}(a)$ decreases monotonically from $\eta^{0}(0)>0$ to $\eta^{0}\left(a_{m}\right)=0$ ). The associated angular velocity $\Omega^{0}$ is bounded $\left(\psi^{0} \sim c Q a / m_{*}\right.$ and $\Omega^{0}(a) \sim c Q / m_{*}=O(1)$ when $a \rightarrow 0$, with $Q \neq 0$ the total charge of the system), and choosing $\Omega_{r}<\inf _{\Pi} \Omega^{0}(a)$ in Eq. (89) results in $k_{2} \leq \mathrm{d} \chi^{0} / \mathrm{d} a \leq K_{2}$ for some $k_{2}>0$ and $K_{2}>0$. Therefore

$-\frac{K_{1}}{k_{2}} \leq \frac{\mathrm{d} \eta^{0}}{\mathrm{~d} \chi^{0}}=\frac{\mathrm{d} \eta^{0} / \mathrm{d} a}{\mathrm{~d} \chi^{0} / \mathrm{d} a} \leq-\frac{k_{1}}{K_{2}}<0$,

and criterion (86) is satisfied: the steady state is nonlinearly stable. Of course, we have the same result for a disk with $\eta^{0}<0$ increasing with $a$. Note that energy conservation does not play a basic role in the argument above, and we could obtain a similar stability property by considering the conserved quantity $G_{1}=-\Omega_{r} J+F$ instead of $G$.

To conclude this section, we give an example of a stable disk. We take $\boldsymbol{B}$ to be dipolar and choose

$\sigma^{0}(r)=\frac{Q_{d} \ell\left(r-r_{*}\right)}{\pi \arctan \left(\ell / r_{*}\right)\left(\ell^{2}+r^{2}\right)^{2}}$.

$\sigma^{0}(r)$ increases from 0 at the star up to some maximum value reached at $r_{m}=\left[\sqrt{4 r_{*}^{2}+\ell^{2}}+2 r_{*}\right] / 3$, and then decreases to 0 (as $r^{-3}$ for large $r$ ). The parameter $\ell \geq 0$ controls the repartition of the total charge $Q_{d}>0$ over $P$. Taking for instance $\ell=0$, we get $r_{m}=4 r_{*} / 3$ and $95 \%$ of $Q_{d}$ is contained inside $r_{95 \%} \sim 40 r_{*}$. The function $\eta^{0}$ associated with $\sigma^{0}$ and $\boldsymbol{B}$ is given by

$\eta^{0}(a)=\frac{Q_{d} \ell\left(1-a / a_{m}\right)}{\pi c a_{m} r_{*} \arctan \left(\ell / r_{*}\right)\left[1+\left(\ell / r_{*}\right)^{2}\left(a / a_{m}\right)^{2}\right]^{2}}$,

and it is checked at once to satisfy the nonlinear stability condition discussed above (for instance, we have $\mathrm{d} \eta^{0} / \mathrm{d} a=$ $-Q_{d} /\left(\pi c a_{m}^{2}\right)$ when $\left.\ell=0\right)$.

\section{Conclusion}

In this paper, we have revisited a simplified model of pulsar electrosphere - first introduced in Pétri et al. (2002b) - in which all the charge-separated plasma outside the pulsar is assumed to be concentrated in an infinitely thin equatorial disk where it moves at the drift velocity in the crossed magnetic and electric fields. First, we have put the equations into a convenient form in which the main field variable is the surface charge density per unit of magnetic flux $\eta$ considered to be a function of the azimuthal angle $\varphi$ and the radial magnetic flux coordinate $a$ (and time $t$ ). We have also shown that, as in most ideal fluid theories, these equations can be given a non-canonical Hamiltonian form by introducing a set of functionals acting on the functions defined on the domain $\Pi$ and a Lie-Poisson bracket. Next, we have proven that a series of global quantities keep a constant value during the evolution of the disk: the integral $F$ of any function $f(\eta)$, the generalized angular momentum $J$ of the disk particles in the magnetic field and an energy $W$ (which is not exactly the total electrostatic energy associated with the introduction of the disk into the surroundings of the star). The model thus admits an infinite number of conservation laws, which are actually the exact counterparts of the ones derived for a laboratory non-neutral plasma confined inside a cylinder by a uniform magnetic field (CNNP) (Davidson 1990; Davidson \& Lund 1991). For a plasma confined inside a torus by a toroidal field (TNNP), the corresponding quantities apart from the angular momentum are also conserved (O’Neil \& Smith 1994).

As an application of the conservation laws, we have derived confinement and stability results. As to our confinement theorems, the first one merely fixes a upper bound (depending on the initial conditions) on the amount of charge which can reach arbitrarily large distances from the pulsar. It is based on angular momentum conservation, and is similar (albeit somewhat more general) to a result recently reported by Pétri et al. (2003) and to an earlier one applying to CNNP due to O'Neil (1980). The second theorem rests on the fact that the surface charge density is frozen in the magnetic field - i.e., the value of $\eta$ attached to an element of plasma keeps a constant value. It is much stronger than the first one, as it implies that it is impossible for a disk which is initially of finite extent to evolve in such a way that a finite amount of charge is expelled to infinity.

Our nonlinear stability theorem concerns a steady state, possibly uniformly rotating, and thus characterized by a density $\eta^{0}$ which is a function of the rotating potential $\chi^{0}$. It implies in particular the stability of an axisymmetric steady state for which $\eta^{0}$ increases with $r$ from 0 at $r_{*}$ up to a constant positive value at infinity. Alternatively, we can take the criterion as providing us with a necessary condition of instability. Our results here are formally identical to those reported in Davidson \& Lund (1991) and Davidson (1990) for CNNP, but they differ from those obtained by O'Neil \& Smith (1994) for TNNP. Clearly, this is due to the fact that we have a law of conservation of angular momentum, which makes our system closer to a CNNP than to a TNNP in spite of the fact that it is threaded by a nonuniform magnetic field.

Let us conclude by a brief remark on a consequence of our work for the building up of a model of the pulsar magnetosphere. A Michel's type electrosphere around an aligned rotator is a dead object, it does not emit either particles or radiation, and there may be only one possibility left to make it active: that it is actually unstable with respect to modes having a development leading to a loss of particles, as hoped by the authors who have most recently introduced the new idea of diocotron instability of an electrosphere (Pétri et al. 2002b, 2003; Spitkovsky \& Arons 2002). What is implied by our second confinement theorem is that, in the absence of particle production, even an unstable equatorial belt is going to stay confined near the pulsar. This shows a contrario the necessity of charges being continuously added to the disk, e.g., by pair production, to obtain a non-negligible flux of particles at large distances - 
a wind -, and to make the sought new picture possibly work. We are currently trying to introduce such a process into the analytical formalism presented in this paper, and we hope to be able to present new results in the near future.

Acknowledgements. I wish to thank J. Heyvaerts for interesting correspondence on the problem of angular momentum conservation.

\section{Appendix A: Non-canonical Hamiltonian structure of the equations}

To build up a non-canonical Hamiltonian formulation of the disk equations, we introduce a space $E$ of functions $\eta(\varphi, a)$ defined on $\Pi$, and a space $\hat{E}$ of functionals defined on $E$, a functional $\mathcal{F} \in \hat{E}$ associating a real number $\mathcal{F}[\eta]$ to $\eta \in E$. The elements of $E$ and those of $\hat{E}$ are assumed to satisfy some regularity assumptions that the reader wanting more mathematical details can find in the review paper by Morrison (1998). A particularly important element in $\hat{E}$ is the Hamiltonian functional $\mathcal{H}$ defined by Eq. (31).

For any $\mathcal{F} \in \hat{E}$, it is possible to construct a so-called functional derivative $\delta \mathcal{F} / \delta \eta$ by setting

$\delta \mathcal{F}[\eta, \delta \eta]=\int_{\Pi} \frac{\delta \mathcal{F}}{\delta \eta} \delta \eta \mathrm{d} \tau$

where $\delta \mathcal{F}[\eta, \delta \eta]$ is the first order variation suffered by $\mathcal{F}[\eta]$ when $\eta$ is replaced by $\eta+\delta \eta$. For instance, $\mathcal{H}$ admits the functional derivative

$$
\frac{\delta \mathcal{H}}{\delta \eta}[\eta \mid \varphi, a]=\int_{\Pi} G \eta^{\prime} \mathrm{d} \tau^{\prime}+\psi_{*}=\psi_{\eta}(\varphi, a),
$$

with the quantity $\psi_{\eta}$ being nothing but the sum of the potential created by the arbitrary $\eta$ (and images) and the star potential.

To any two functionals $\mathcal{F}$ and $\mathcal{G}$ in $\hat{E}$ there is associated the Lie-Poisson bracket $\{\mathcal{F}, \mathcal{G}\}$ defined by Eq. (30), in which appear the functional derivatives $\delta \mathcal{F} / \delta \eta$ and $\delta \mathcal{G} / \delta \eta$. This bracket is obviously antisymmetric, i.e.,

$$
\{\mathcal{G}, \mathcal{F}\}=-\{\mathcal{F}, \mathcal{G}\}
$$

and it does satisfy the Jacobi identity

$$
\{\{\mathcal{E}, \mathcal{F}\}, \mathcal{G}\}+\{\{\mathcal{G}, \mathcal{E}\}, \mathcal{F}\}+\{\{\mathcal{F}, \mathcal{G}\}, \mathcal{E}\}=0
$$

(The proof of the latter is similar to the one given in Morrison 1998, for the bracket used in the Hamiltonian description of a two-dimensional incompressible fluid).
Time does not intervene in the formalism presented above, and we reintroduce it now by considering a solution $(\eta(t), \psi(t))$ describing a physical evolution of the disk (then $\psi=\psi_{\eta}$ at any time). Choosing an arbitrary functional $\mathcal{F}$ not depending explicitly on time, we have

$$
\begin{aligned}
\frac{\mathrm{d}}{\mathrm{d} t} \mathcal{F}[\eta(t)] & =\int_{\Pi} \frac{\delta \mathcal{F}}{\delta \eta} \frac{\partial \eta}{\partial t} \mathrm{~d} \tau \\
& =\int_{\Pi}[\psi, \eta] \frac{\delta \mathcal{F}}{\delta \eta} \mathrm{d} \tau \\
& =\int_{\Pi}\left[\frac{\delta \mathcal{F}}{\delta \eta}, \frac{\delta \mathcal{H}}{\delta \eta}\right] \eta \mathrm{d} \tau,
\end{aligned}
$$

where we have used formula (35) (with $f=\psi$ independent of $\varphi$ on $\Lambda_{0}$ and $\Lambda$ ). Taking into account the definition of $\{.,$.$\} ,$ we thus obtain Eq. (32). Reciprocally, it is easily checked that if Eq. (32) holds true for any functional $\mathcal{F}$, then $\left(\eta(t), \psi_{\eta}(t)\right)$ solves the evolution problem for the disk.

In this formalism, conservation laws are associated with functionals $C$ - the so-called Casimirs - which commute with $\mathcal{H}$, i.e.,

$\{C, \mathcal{H}\}=0$,

a property which depends only on the structure of $C$. By using in particular Eq. (35), it is possible to show that the functionals $\mathcal{F}$ and $\mathcal{J}$ defined by

$$
\begin{aligned}
& \mathcal{F}[\eta]=\int_{\Pi} f(\eta) \mathrm{d} \tau, \\
& \mathcal{J}[\eta]=\int_{\Pi} a \eta \mathrm{d} \tau,
\end{aligned}
$$

and, in a quite obvious way, the Hamiltonian functional $\mathcal{H}$ itself, are all Casimirs, the associated conservation laws being just the ones we have derived in a more elementary way in Sect. 5. It is worth noticing the very direct way in which energy conservation is included in the formalism.

\section{References}

Aly, J. J. 1994, Phys. Plasmas, 1, 2401

Aly, J. J. 2005, A\&A, 429, 779

Davidson, R. C. 1990, Physics of nonneutral plasmas (AddisonWesley Publishing Company)

Davidson, R. C., \& Lund, S. M. 1991, Phys. Fluids B, 3, 2540

Goldreich, P., \& Julian, W. H. 1969, ApJ, 157, 869

Holm, D. H., Marsden, J. E., Ratiu, T., \& Weinstein, A. 1985, Phys. Rep., 123, 1

Jackson, J. D. 1975, Classical Electrodynamics (New York: Wiley)

Krause-Polstorff, J., \& Michel, F. C. 1985a, A\&A, 144, 72

Krause-Polstorff, J., \& Michel, F. C. 1985b, MNRAS, 213, 43

Michel, F. C. 1980, Ap\&SS, 72, 175

Morrison, P. J. 1998, Rev. Mod. Phys., 70, 467

O'Neil, T. 1980, Phys. Fluids, 23, 2216

O’Neil, T., \& Smith, R. A. 1994, Phys. Plasmas, 1, 2430

Pétri, J., Heyvaerts, J., \& Bonazzola, S. 2002a, A\&A, 384, 414

Pétri, J., Heyvaerts, J., \& Bonazzola, S. 2002b, A\&A, 387, 520

Pétri, J., Heyvaerts, J., \& Bonazzola, S. 2003, A\&A, 411, 203

Smith, I. A., Michel, F. C., \& Thacker, P. D. 2001, MNRAS, 322, 209

Spitkovsky, A., \& Arons, J. 2002 [arXiv: astro-ph/0201360] 